\title{
MGD: Definition Versus Dry Eye Disease, Risk Factors
}

\author{
Anat Galor
}

Published online: 1 April 2014

(c) Springer Science + Business Media New York 2014

\begin{abstract}
Meibomian gland dysfunction (MGD) is defined as any functional disorder of the meibomian glands. While MGD is commonly associated with lipid tear deficiency, patients with MGD may also be asymptomatic and/or may have normal tear film parameters. Although there are no standardized diagnostic criteria for the disease, epidemiologic studies have revealed that MGD is very common, especially in Asian populations. Risk factors for MGD include both ocular and systemic conditions. Ocular conditions specifically found to associate with MGD include contact lens wear and the use of glaucoma medications. Systemically, older age, lower androgen levels, rosacea, and Sjogren's syndrome have all been associated with disease.
\end{abstract}

Keywords Meibomian gland dysfunction - Lipid tear deficiency · Contact lens wear · Glaucoma medication . Rocasea · Androgen deficiency · Nutrition · Sjogren's syndrome

\section{Introduction}

Meibomian Glands (MG)

The meibomian glands (MGs) are a subtype of sebaceous glands located in the eyelid. They differ from other

A. Galor $(\bowtie)$

Miami VA Medical Center, 1201 NW 16th Street, Miami, FL

33125, USA

e-mail: agalor@med.miami.edu

A. Galor

Bascom Palmer Eye Institute, University of Miami, 900 NW

17th Street, Miami, FL 22126, USA sebaceous glands as they do not associate with hair follicles. The MGs are composed of clusters of secretory acini connected to a central duct by short ductules. Their central duct openings are found in a single file line in the posterior part of the upper and lower eyelid margins, anterior to the mucocutaneous junction. Meibomian acini, consistent with other sebaceous glands, release lipid by holocrine secretion. That is, the cell membrane of a mature meibocyte disintegrates, and the cell content is released to form a lipid product called meibum [1••]. Meibum is a complex substance consisting of nonpolar lipids (e.g., cholesterol, cholesterol esters, wax esters) and polar lipids (e.g., phospholipids) of varying concentrations [2••]. Meibum comprises the superficial layer of the tear film and protects against its evaporation. Healthy MG function is needed to maintain a healthy tear film and ocular surface.

\section{Meibomian Gland Dysfunction (MGD)}

MGD is defined as any functional disorder of the MGs. This includes low delivery states due to hypo-secretion or obstruction and high delivery states due to hyper-secretion [3]. It is challenging to study the epidemiology of MGD as there are no standardized diagnostic criteria to define this entity. MG dysfunction can be evaluated by a combination of static and dynamic tests performed at the slit lamp (Table 1). Static tests include the grading of eyelid margin telangiectasias (Fig. 1), keratinization, MG plugging, and MG foaminess. Dynamic tests include the grading of meibum quality and expressibility. The lipid product in patients with MGD is typically thicker than normal meibum and is more difficult or impossible to express.

In addition to these simple metrics, other tests, both commercially and non-commercially available, can be applied to indirectly evaluate MG function. LipiView (Tear 
Table 1 Commonly used tests to evaluate MGD

Slit lamp-based tests
Static tests
Lid margin telangiectasias
Lid margin keratinization
MG orifice plugging
MG orifice foaminess
Dynamic tests
Tear break-up time
MG expressibility
Determination of meibum quality
Commercially available tests
Interferometer (LipiView): quantifies thickness of lipid tear film
layer
Confocal imaging (Heidelberg Retina Tomograph II-Rostock
Cornea Module): allows assessment of MG morphology
Non-commercially available tests
Evaporimetry
Meibography (may be performed with tranilluminator and
visually graded or images may be recorded and subsequently
analyzed): allows assessment of MG morphology/dropout

$M G D$ meibomian gland dysfunction, $M G$ meibomian glands

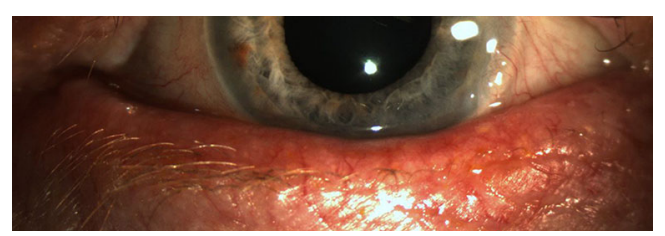

Fig. 1 Slit lamp photograph demonstrating telangiectasias of the lower eyelid margin, one of the many diagnostic tests that can be used to evaluate for the presence of MGD

Science, Morrisville, NC) is a commercially available interferometer that is packaged with the LipiFlow Thermal Pulsation System. LipiView quantifies the thickness of the lipid tear film layer, and information on lipid spreading and stability can also be non-quantitatively evaluated in the generated images. In vivo laser scanning confocal microscopy (Heidelberg Retina Tomograph II-Rostock Cornea Module; Heidelberg Engineering GmbH, Dossenheim, Germany) is another commercially available product that can be used to study the morphology of the MG [4••]. Examined metrics in MGD include the longest and shortest acinar unit diameter, periglandular inflammatory cell density, and acinar unit density.

Evaporimetry is a non-commercially available test that measures tear evaporation from the ocular surface. Estimates of evaporation rates have been generated in the research arena by studying pressure and humidity gradients using a variety of techniques [4••]. Meibography is a technique that can easily be integrated into clinical practice by applying a transilluminator to the external skin while the lower lid is everted. By retroilluminating the MGs, morphology and dropout can be assessed. A more developed research tool records the images allowing for more detailed structural evaluation of the MGs.

Despite the availability of so many metrics and tests, there is no agreement on which one or combination of findings should define MGD. As such, different studies have used different measures to define the disease and report on its epidemiology. To add to the confusion, abnormalities in any of the above measures can be found with or without ocular surface symptoms, ocular surface damage (i.e., corneal and conjunctival staining) or abnormal tear evaporation. These limitations must be kept in mind when evaluating the literature regarding prevalence and risk factors for MGD.

\section{Epidemiology of MGD}

\section{Prevalence of MGD}

Various population-based studies have used various clinical measures to study MGD prevalence (Table 2). The Beijing Eye Study, for example, used the presence of telangiectasias at the lid margin (yes/no) to define MGD and found an overall prevalence of $68 \%(n=1,336 / 1,957)$ [5]. Other studies out of Asia similarly found high frequencies of eyelid abnormalities with $46 \%(n=254 / 550)$ of Thai individuals $>40$ years having telangietasias, MG plugging or collarettes [6], and $62 \%(n=70 / 113)$ of Japanese individuals $>60$ years having gland dropout and abnormal MG quality [7]. A USA population-based study, on the other hand, evaluated 2,482 white individuals over the age of 65 in Maryland and found that $3.5 \%$ had grade 2 or higher MG plugging or collarettes. A study out of Spain used a different disease definition, namely viscous, waxy or no secretion upon expression, lid margin telangiectasia or MG plugging and found a $31 \%$ frequency of MGD in their population.

Based on the limitations stated above, however, it is difficult to compare MGD prevalence estimates between studies because of differences in the population studied (e.g., different age cutoffs, inclusion methodology) and differences in the clinical definitions of disease.

Prevalence of Lipid Versus Aqueous Tear Deficiency (ATD)

Lipid tear deficiency (LTD) is defined as tear dysfunction in the setting of an abnormal lipid tear layer. It is most commonly assessed by non-invasively measuring the tear break-up time (TBUT), although other tests can also be employed including 
Table 2 Frequency of MGD in different populations

\begin{tabular}{|c|c|c|c|c|c|c|c|}
\hline & Author & Year & $\mathrm{N}$ & Location & Population & Definition of MGD & Frequency $(\%)$ \\
\hline \multirow[t]{6}{*}{ Asia } & $\operatorname{Lin}^{\mathrm{a}}$ & 2003 & 1,361 & Shihpai, Taiwan & Population-based & $\begin{array}{l}\text { Telangiectasia or MG orifice } \\
\text { plugging }\end{array}$ & 61 \\
\hline & Lekhanont $^{\mathrm{b}}$ & 2006 & 550 & Bangkok, Thailand & Population-based & $\begin{array}{l}\text { Telangiectasia, MG orifice } \\
\text { plugging or collarettes }\end{array}$ & 46 \\
\hline & Uchino $^{c}$ & 2006 & 113 & Near Tokyo, Japan & Population-based & $\begin{array}{l}\text { Gland dropout, poor MG } \\
\text { expressibility or poor meibum } \\
\text { quality }\end{array}$ & 62 \\
\hline & $\mathrm{Jie}^{\mathrm{d}}$ & 2009 & 1,957 & Beijing, China & Population-based & Telangiectasia & 68 \\
\hline & $\operatorname{Siak}^{\mathrm{e}}$ & 2012 & 3,271 & Singapore & Population-based & $\begin{array}{l}\text { Telangiectasia or MG orifice } \\
\text { plugging }\end{array}$ & 56 \\
\hline & Basak $^{\mathrm{f}}$ & 2012 & 3,023 & West Bengal, India & Clinic-based & Poor meibum quality & 32 \\
\hline \multirow[t]{3}{*}{ USA } & Schein $^{\mathrm{g}}$ & 1997 & 2,482 & Salisbury, Maryland & Population-based & MG orifice plugging or collarettes & 4 \\
\hline & Hom $^{\mathrm{h}}$ & 2005 & 398 & Southern California & Hispanics, Clinic based & Poor meibum quality & 39 \\
\hline & Galor $^{\mathrm{i}}$ & 2013 & 263 & Miami, Florida & Men, Clinic-based & $\begin{array}{l}\text { MG orifice plugging or poor } \\
\text { meibum quality }\end{array}$ & 59 \\
\hline Europe & Viso $^{\mathrm{j}}$ & 2011 & 654 & O Salnés, Spain & Population-based & $\begin{array}{l}\text { Telangiectasia, MG orifice } \\
\text { plugging or poor meibum quality }\end{array}$ & 31 \\
\hline
\end{tabular}

$M G D$ meibomian gland dysfunction, $M G$ meibomian gland

${ }^{a} \operatorname{Ref}[56]$

b Ref. [6]

c Ref. [7]

${ }^{d}$ Ref. [5]

e Ref. [46]

${ }^{f}$ Ref. [57]

${ }^{\mathrm{h}}$ Ref. [58]

${ }^{i}$ Ref. [59]

j Ref. [10॰]

${ }^{k}$ Ref. [60]

interferometry and evaporimetry [4••]. This finding can be seen in patients with MGD, but not all patients with MGD have LTD. LTD must be differentiated from ATD, which is defined as tear dysfunction in the setting of an abnormal aqueous tear layer. Studies have shown that isolated LTD and a mixed pattern of LTD and ATD are more common than isolated ATD [8, 9•]. In a study by Galor et al. [10•], 176 of 263 men were found to have DES by objective tear film parameters. Of these, $22(8 \%)$ had mostly ATD, 124 (47 \%) mostly LTD and $30(11 \%)$ a mixed pattern.

\section{Symptoms in MGD}

It is well known that there is poor correlation between signs and symptoms in DES [10, 11]. MGD is no exception, and many patients with clinically apparent MGD have been found to be asymptomatic [12•]. In the Beijing Eye Study, $79 \%$ ( $n=1,051 / 1,336)$ with MGD (i.e., presence of lid telangiectasias) had no DES symptoms [5]. Similarly, in the Bangkok Study, $54 \%(n=136 / 254)$ with MGD (i.e., telangietasias, MG plugging or collarettes) were asymptomatic [6]. Conversely, in a
US Veterans Administration (VA) eye clinic-based study, only $51 \%(n=63 / 124)$ with LTD and $57 \%(n=17 / 30)$ with a mixed pattern complained of severe DES symptoms as assessed by the dry eye questionnaire 5 (DEQ5) [10•].

\section{MGD Risk Factors}

Few studies have specifically evaluated for MGD-associated risk factors and have instead focused on DES-associated risk factors, incorporating both the ATD and LTD forms of disease. However, some ocular and systemic risk factors have specifically been associated with MGD, and these will be described below.

\section{Ocular Risk Factors for MGD}

\section{Contact Lens (CL) Wear}

There is a clinical impression that CL wearers have a higher risk of MGD compared to similarly aged non-CL 
wearers. However, limited data are available on the impact of CL wear on the MGs. In one study of 121 individuals, CL wearers had significantly more MG changes than agematched controls, and these changes increased with longer duration of CL wear [13]. In another, evidence of MGD was found in $8.6 \%$ of 4,064 soft CL wearers in BP Koirala Lions Centre for Ophthalmic Studies in Kathmandu, Nepal [14]. No control group, however, was provided for comparison.

\section{Topical Medications}

Epinephrine eyedrops, now no longer clinically utilized, have been described to affect MG function [1••]. Glaucoma medications in general have also been found to affect MG morphology with lower acinar density and area in those using two or more glaucoma medications compared to those not on medications [15]. Patients using any medication also had less homogeneous acinar wall morphology compared to those not on glaucoma drops. Preserved prostaglandin analogs were associated with significantly more morphologic abnormalities in all measured values compared to non-preserved products. Clinically, patients on glaucoma drops were found to have higher meibum scores and lower TBUT than those not on drops [16].

\section{Ocular Surface Microbiome}

The ocular surface microbiome may influence the quality of meibum as secreted lipids may be modified by hydrolyzing enzymes from commensal bacteria inside the ductal system and on the ocular surface. These enzymes may have the effect of breaking down triglycerides into free fatty acids [17]. Free fatty acids, subsequently, may be one of the drivers of the hyper-keratinization seen in MGD [1・•]. Demodex infestation is another ocular factor postulated to affect the MGs. In a study of six patients with Demodex, five had evidence of MGD [18]. Additionally, other studies have linked Demodex to rocasea, a disease independently associated with MGD [19, 20].

\section{Systemic Risk Factors for MGD}

Age

A cross-sectional study demonstrated an increased frequency of eyelid margin abnormalities (e.g., vascularity, keratinization) in older compared to younger individuals [21]. Other studies have supported this observation by demonstrating concomitant changes in meibum quality with both polar and neutral lipid profiles being affected with age [22, 23]. On a cellular level, acinar epithelial cells of the MG have been found to undergo atrophy with age, with a concomitant decrease in lipid production [1••]. These changes are suspected to occur in the setting of declining hormone levels that occur with age.

\section{Hormones}

Androgens are known to affect sebaceous glands throughout the body $[1 \bullet \cdot$. As such, MGs have also been shown to be influenced by hormonal status. Meibomian acini harbor androgen and estrogen receptors $[1 \bullet \bullet, 24 \bullet \bullet, 25$, 26], and they contain the enzymes necessary for the intracrine synthesis and metabolism of sex steroids (5alphareductase, aromatase, 17-hydroxysteroid dehydrogenase) $[1 \bullet, 24 \bullet \bullet, 27]$. Schirra et al. found that testosterone influenced the expression of over 1,500 genes in MG tissue from orchiectomized mice. Pathways affected included those of lipid metabolism, sterol biosynthesis, and fatty acid metabolism [28•]. Khandelwal et al. [29] found similar results in an immortalized human MG cell line as 3,000 genes were influenced by the addition of DHT to the culture. Orchiectomy has been shown to change the lipid pattern in rabbit MG with subsequent normalization after administration of 19-nortestosterone [30].

Clinically, MGD has been described in various androgen-depleted states including men receiving anti-androgen therapy (e.g., for prostate cancer), women with congenital androgen insensitivity syndrome, and Sjogren's syndrome [24••, 31-33]. Men on anti-androgen therapy have been found to have abnormal MG secretions with an altered lipid profile [32]. Men and women with MGD and DES have also been found to have lower bioavailable $\mathrm{T}$ and dehydroepiandrosterone (DHEA) levels compared to non-MGD dry eye patients and controls [34].

Limited data are available, however, on whether oral or topical hormonal supplementation should be used in patients with MGD and DES. Several trials found that while oral dehydroepiandrosterone (DHEA) improved dry mouth and constitutive symptoms, it did not affect dry eye symptoms in Sjogren's syndrome [35-37]. In one case report, topical androgen therapy improved the tear lipid layer in a patient with MGD [38]. Systemic estrogen, with or without progesterone therapy, on the other hand, has been found to have either no or an adverse effect on DES $[1 \bullet \bullet$.

\section{Rosacea}

The link between rosacea and MGD has been well described [39*•]. In one study, 60,042 rosacea cases and 60,042 controls were identified using the UK-based General Practice Research Database. The overall incidence rate for diagnosed rosacea was 1.65 per 1,000 person-years. Ocular symptoms were documented in $21 \%$ of cases at the 
time of diagnosis $\left[40^{\bullet}\right]$. Another study reported that $33 \%$ of 100 rosacea patients in Northern Greece had evidence of ophthalmic involvement in the form of symptoms or signs of disease (e.g., burning, blepharitis, conjunctivitis). Furthermore, patients with rosacea had significantly lower TBUT than age-matched controls [41].

\section{Systemic Medications}

Retinoic acid (RA), a metabolite of vitamin A, has been used for the treatment of various dermatologic disorders. MGD has been well described in the setting of systemic 13-cis RA (isotretinoin) use [42, 43]. For example, the MGs of 11 patients treated with isotretinoin were assessed before and during treatment. The MGs appeared more atrophic by meibography; meibum thickness increased and meibum volume decreased during treatment [44]. In another study of 40 patients treated with isotretinoin for 2 months, 20 patients $(50 \%)$ had a decrease in TBUT to less than $10 \mathrm{~s}$ and $22(55 \%)$ had newly documented blepharitis. Fortunately, all ocular abnormalities disappeared 1 month after cessation of treatment [42]. A recent study shed light on these observations and demonstrated that 13-cis RA inhibited proliferation, increased cell death, and increased inflammatory mediator expression in immortalized human MG epithelial cells [45]. Other medications that have been specifically associated with MGD include angiotensin II receptor blockers [46 ${ }^{\circ}$, antiandrogens, and hormone replacement therapy [39••].

\section{Nutrition}

There is much interest in understanding how diet influences MG function and DES. Studies have documented qualitative lipid changes in patients with MGD, including a decrease in monounsaturated fatty acids and polar lipids [1••]. Epidemiological evidence points to the protective effect of essential fatty acids on DES. In a cross-sectional study of over 32,000 women, higher dietary intake of fish oil and omega-3 was associated with a decreased incidence of DES [47]. In clinical trials, oral supplementation of omega-3 improved tear film parameters including TBUT and decreased ocular surface inflammation (as measured by HLA-DR) in patients with DES [48, 49••, 50]. One study of women with Sjogren's syndrome found a difference in polar lipid profiles based on intake of omega-3 [51]. However, a study of patients with blepharitis taking flaxseed oil failed to show a difference in meibum properties (e.g., omega-3 and 6 fatty acids) despite levels of omega-3 being higher in blood [50]. This study, however, reported that the quality of meibum was clinically improved with treatment.

\section{Sjogren's Syndrome}

Traditionally, SS has been thought of as a pure ATD form of DES. Studies have demonstrated, however, that patient's with SS have a higher frequency of severe MG obstruction [52] and higher tear evaporation [53] compared to non-SS aqueous tear-deficiency individuals. It is unclear whether these changes are a primary consequence of disease or whether this represents a secondary change. Similar findings have been described in other ATD-specific diseases such as graft versus host disease (GVHD) [54]. GVHD patients have been found to have higher MG dropout [54] and lower MG acinar density and diameter [55] than control patients without GVHD. In general, increased lipid layer spreading abnormalities have been described in patients with increasing ATD severity [39••].

\section{Conclusion}

MGD is a common finding in patients with and without symptoms of DES. While much still remains to be elucidated about the pathophysiology and natural history of disease, epidemiological data regarding risk factors suggest that ocular factors (e.g., contact lens use) and systemic factors (e.g., hormonal status and nutrition) play a role in the health of the MGs and in disease.

\section{References}

Papers of particular interest, published recently, have been highlighted as:

- Of importance

•• Of major importance

1. •- Knop E, Knop N, Millar T, Obata H, Sullivan DA. The international workshop on meibomian gland dysfunction: report of the subcommittee on anatomy, physiology, and pathophysiology of the meibomian gland. Invest Ophthalmol Vis Sci. 2011;52:1938-78. Landmark set of reviews on meibomian gland dysfunction

2. •• Foulks GN, Bron AJ. Meibomian gland dysfunction: a clinical scheme for description, diagnosis, classification, and grading. Ocul Surf. 2003;1:107-26. Landmark set of reviews on meibomian gland dysfunction

3. Nichols KK, Foulks GN, Bron AJ, et al. The international workshop on meibomian gland dysfunction: executive summary. Invest Ophthalmol Vis Sci. 2011;52:1922-9.

4. • Tomlinson A, Bron AJ, Korb DR, et al. The international workshop on meibomian gland dysfunction: report of the diagnosis subcommittee. Invest Ophthalmol Vis Sci. 2011;52:2006-49. Landmark set of reviews on meibomian gland dysfunction 
5. Jie Y, Xu L, Wu YY, Jonas JB. Prevalence of dry eye among adult Chinese in the Beijing Eye Study. Eye (Lond). 2009;23: 688-93.

6. Lekhanont K, Rojanaporn D, Chuck RS, Vongthongsri A. Prevalence of dry eye in Bangkok, Thailand. Cornea. 2006;25: 1162-7.

7. Uchino M, Dogru M, Yagi Y, et al. The features of dry eye disease in a Japanese elderly population. Optom Vis Sci. 2006;83:797-802.

8. Shimazaki J, Sakata M, Tsubota K. Ocular surface changes and discomfort in patients with meibomian gland dysfunction. Arch Ophthalmol. 1995;113:1266-70.

9. - Lemp MA, Crews LA, Bron AJ, Foulks GN, Sullivan BD. Distribution of aqueous-deficient and evaporative dry eye in a clinic-based patient cohort: a retrospective study. Cornea. 2012;31:472-8. Study demonstrating that evaporative dry eye resulting from MGD is much more prevalent than pure ATD in a clinic-based patient population

10. - Galor A, Feuer W, Lee DJ, Florez H, Venincasa VD, Perez VL. Ocular surface parameters in older male veterans. Invest Ophthalmol Vis Sci. 2013;54:1426-33. Study in older male patients demonstrating a lack of correlation between symptoms and signs of dry eye disease, including in those with LTD

11. Schein OD, Tielsch JM, Munoz B, Bandeen-Roche K, West S. Relation between signs and symptoms of dry eye in the elderly. A population-based perspective. Ophthalmology. 1997;104: $1395-401$.

12. - Viso E, Rodriguez-Ares MT, Abelenda D, Oubina B, Gude F. Prevalence of asymptomatic and symptomatic meibomian gland dysfunction in the general population of Spain. Invest Ophthalmol Vis Sci. 2012;53:2601-6. Study demonstrating that asymptomatic MGD is more common than symptomatic MGD

13. Arita R, Itoh K, Inoue K, Kuchiba A, Yamaguchi T, Amano S. Contact lens wear is associated with decrease of meibomian glands. Ophthalmology. 2009;116:379-84.

14. Sapkota K, Lira M, Martin R, Bhattarai S. Ocular complications of soft contact lens wearers in a tertiary eye care centre of Nepal. Cont Lens Anterior Eye. 2013;36:113-7.

15. Agnifili L, Fasanella V, Costagliola C, et al. In vivo confocal microscopy of meibomian glands in glaucoma. Br J Ophthalmol. 2013;97:343-9.

16. Arita $\mathrm{R}$, Itoh $\mathrm{K}$, Maeda $\mathrm{S}$, et al. Comparison of the long-term effects of various topical antiglaucoma medications on meibomian glands. Cornea. 2012;31:1229-34.

17. Dougherty JM, McCulley JP. Bacterial lipases and chronic blepharitis. Invest Ophthalmol Vis Sci. 1986;27:486-91.

18. Kheirkhah A, Casas V, Li W, Raju VK, Tseng SC. Corneal manifestations of ocular demodex infestation. Am J Ophthalmol. 2007;143:743-9.

19. Zhao YE, Peng Y, Wang XL, et al. Facial dermatosis associated with Demodex: a case-control study. J Zhejiang Univ Sci B. 2011;12:1008-15.

20. Zhao YE, Wu LP, Peng Y, Cheng H. Retrospective analysis of the association between Demodex infestation and rosacea. Arch Dermatol. 2010;146:896-902.

21. Hykin P, Bron A. Age-related morphological changes in lid margin and meibomian gland anatomy. Cornea. 1992;11:334-42.

22. Sullivan BD, Evans JE, Dana MR, Sullivan DA. Impact of androgen deficiency on the lipid profiles in human meibomian gland secretions. Adv Exp Med Biol. 2002;506:449-58.

23. Sullivan BD, Evans JE, Dana MR, Sullivan DA. Influence of aging on the polar and neutral lipid profiles in human meibomian gland secretions. Arch Ophthalmol. 2006;124:1286-92.

24. - Sullivan DA, Sullivan BD, Evans JE, et al. Androgen deficiency, meibomian gland dysfunction, and evaporative dry eye. Ann N Y Acad Sci. 2002;966:211-22. Comprehensive study evaluating the role of androgens on the meibomian gland in animal models and in humans

25. Auw-Haedrich C, Feltgen N. Estrogen receptor expression in meibomian glands and its correlation with age and dry-eye parameters. Graefes Arch Clin Exp Ophthalmol. 2003;241:705-9.

26. Esmaeli B, Harvey JT, Hewlett B. Immunohistochemical evidence for estrogen receptors in meibomian glands. Ophthalmology. 2000;107:180-4.

27. Rocha EM, Wickham LA, da Silveira LA, et al. Identification of androgen receptor protein and 5alpha-reductase mRNA in human ocular tissues. Br J Ophthalmol. 2000;84:76-84.

28. - Schirra F, Suzuki T, Richards SM, et al. Androgen control of gene expression in the mouse meibomian gland. Invest Ophthalmol Vis Sci. 2005;46:3666-75. Study demonstrating that androgens affect the expression of more than 1500 genes in mouse meibomian glands

29. Khandelwal P, Liu S, Sullivan DA. Androgen regulation of gene expression in human meibomian gland and conjunctival epithelial cells. Mol Vis. 2012;18:1055-67.

30. Sullivan DA, Sullivan BD, Ullman MD, et al. Androgen influence on the meibomian gland. Invest Ophthalmol Vis Sci. 2000;41: 3732-42.

31. Sullivan DA, Belanger A, Cermak JM, et al. Are women with Sjogren's syndrome androgen-deficient? J Rheumatol. 2003;30: 2413-9.

32. Krenzer KL, Dana MR, Ullman MD, et al. Effect of androgen deficiency on the human meibomian gland and ocular surface. J Clin Endocrinol Metab. 2000;85:4874-82.

33. Cermak JM, Krenzer KL, Sullivan RM, Dana MR, Sullivan DA. Is complete androgen insensitivity syndrome associated with alterations in the meibomian gland and ocular surface? Cornea. 2003;22:516-21.

34. Tamer C, Oksuz H, Sogut S. Androgen status of the nonautoimmune dry eye subtypes. Ophthalmic Res. 2006;38:280-6.

35. Pillemer SR, Brennan MT, Sankar V, et al. Pilot clinical trial of dehydroepiandrosterone (DHEA) versus placebo for Sjogren's syndrome. Arthritis Rheum. 2004;51:601-4.

36. Hartkamp A, Geenen R, Godaert GL, et al. Effect of dehydroepiandrosterone administration on fatigue, well-being, and functioning in women with primary Sjogren syndrome: a randomised controlled trial. Ann Rheum Dis. 2008;67:91-7.

37. Forsblad-d'Elia H, Carlsten H, Labrie F, Konttinen YT, Ohlsson C. Low serum levels of sex steroids are associated with disease characteristics in primary Sjogren's syndrome; supplementation with dehydroepiandrosterone restores the concentrations. J Clin Endocrinol Metab. 2009;94:2044-51.

38. Worda C, Nepp J, Huber JC, Sator MO. Treatment of keratoconjunctivitis sicca with topical androgen. Maturitas. 2001;37:209-12.

39. • Schaumberg DA, Nichols JJ, Papas EB, Tong L, Uchino M, Nichols KK. The international workshop on meibomian gland dysfunction: report of the subcommittee on the epidemiology of, and associated risk factors for MGD. Invest Ophthalmol Vis Sci. 2011;52:1994-2005. Landmark set of reviews on meibomian gland dysfunction

40. - Spoendlin J, Voegel JJ, Jick SS, Meier CR. A study on the epidemiology of rosacea in the U.K. $\mathrm{Br} \mathrm{J}$ Dermatol. 2012;167:598-605. A large epidemiological study that reported on the frequency of ocular symptoms in patients with rosacea

41. Lazaridou E, Fotiadou C, Ziakas NG, Giannopoulou C, Apalla Z, Ioannides D. Clinical and laboratory study of ocular rosacea in northern Greece. J Eur Acad Dermatol Venereol. 2011;25: 1428-31.

42. Bozkurt B, Irkec MT, Atakan N, Orhan M, Geyik PO. Lacrimal function and ocular complications in patients treated with systemic isotretinoin. Eur J Ophthalmol. 2002;12:173-6. 
43. Kremer I, Gaton DD, David M, Gaton E, Shapiro A. Toxic effects of systemic retinoids on meibomian glands. Ophthalmic Res. 1994;26:124-8.

44. Mathers WD, Shields WJ, Sachdev MS, Petroll WM, Jester JV. Meibomian gland morphology and tear osmolarity: changes with accutane therapy. Cornea. 1991;10:286-90.

45. Ding J, Kam WR, Dieckow J, Sullivan DA. The influence of 13-cis retinoic acid on human meibomian gland epithelial cells. Invest Ophthalmol Vis Sci. 2013;54:4341-50.

46. - Siak JJ, Tong L, Wong WL, et al. Prevalence and risk factors of meibomian gland dysfunction: the Singapore Malay eye study. Cornea. 2012;31:1223-8. A study which reported that over $50 \%$ of the studied population in Singapore had MGD; associated risk factors included male gender, postmenopausal status, pinguecula, high diastolic blood pressure, and use of angiotensin II receptor blockers

47. Miljanovic B, Trivedi KA, Dana MR, Gilbard JP, Buring JE, Schaumberg DA. Relation between dietary n-3 and n- 6 fatty acids and clinically diagnosed dry eye syndrome in women. Am J Clin Nutr. 2005;82:887-93.

48. Kangari H, Eftekhari MH, Sardari S, et al. Short-term consumption of oral omega-3 and dry eye syndrome. Ophthalmology. 2013;120:2191.

49. •• Brignole-Baudouin F, Baudouin C, Aragona P, et al. A multicentre, double-masked, randomized, controlled trial assessing the effect of oral supplementation of omega- 3 and omega- 6 fatty acids on a conjunctival inflammatory marker in dry eye patients. Acta Ophthalmol. 2011;89:e591-7. Large, multi-center trial evaluating the effect of oral omea-3 and -6 in patients with dry eye disease

50. Macsai MS. The role of omega-3 dietary supplementation in blepharitis and meibomian gland dysfunction (an AOS thesis). Trans Am Ophthalmol Soc. 2008;106:336-56.
51. Sullivan BD, Cermak JM, Sullivan RM, et al. Correlations between nutrient intake and the polar lipid profiles of meibomian gland secretions in women with Sjogren's syndrome. Adv Exp Med Biol. 2002;506:441-7.

52. Shimazaki J, Goto E, Ono M, Shimmura S, Tsubota K. Meibomian gland dysfunction in patients with Sjogren syndrome. Ophthalmology. 1998;105:1485-8.

53. Goto E, Matsumoto Y, Kamoi M, et al. Tear evaporation rates in Sjogren syndrome and non-Sjogren dry eye patients. Am J Ophthalmol. 2007;144:81-5.

54. Khanal S, Tomlinson A. Tear physiology in dry eye associated with chronic GVHD. Bone Marrow Transplant. 2012;47:115-9.

55. Ban Y, Ogawa Y, Ibrahim OM, et al. Morphologic evaluation of meibomian glands in chronic graft-versus-host disease using in vivo laser confocal microscopy. Mol Vis. 2011;17:2533-43.

56. Lin PY, Tsai SY, Cheng CY, Liu JH, Chou P, Hsu WM. Prevalence of dry eye among an elderly Chinese population in Taiwan: the Shihpai Eye Study. Ophthalmology. 2003;110: 1096-101.

57. Basak SK, Pal PP, Basak S, Bandyopadhyay A, Choudhury S, Sar S. Prevalence of dry eye diseases in hospital-based population in West Bengal, Eastern India. J Indian Med Assoc. 2012;110: 789-94.

58. Schein OD, Munoz B, Tielsch JM, Bandeen-Roche K, West S. Prevalence of dry eye among the elderly. Am J Ophthalmol. 1997; $124: 723-8$

59. Hom M, De Land P. Prevalence and severity of symptomatic dry eyes in Hispanics. Optom Vis Sci. 2005;82:206-8.

60. Viso E, Gude F, Rodriguez-Ares MT. The association of meibomian gland dysfunction and other common ocular diseases with dry eye: a population-based study in Spain. Cornea. 2011; $30: 1-6$. 